\title{
Erratum to: Soluble epoxide hydrolase inhibitor 1-trifluoromethoxyphenyl-3-(1-propionylpiperidin-4-yl) urea attenuates bleomycin-induced pulmonary fibrosis in mice
}

\author{
Yong Zhou ${ }^{1}$ · Jun Yang ${ }^{2}$ - Guo-Ying Sun ${ }^{1}$ Tian Liu ${ }^{1}$ - Jia-Xi Duan ${ }^{1} \cdot$ Hui-Fang Zhou ${ }^{1}$. \\ Kin Sing Lee ${ }^{2} \cdot$ Bruce D. Hammock $^{2} \cdot$ Xiang Fang $^{3} \cdot$ Jian-Xin Jiang ${ }^{4} \cdot$ Cha-Xiang Guan ${ }^{1}$
}

Published online: 5 March 2016

(C) Springer-Verlag Berlin Heidelberg 2016

Erratum to: Cell Tissue Res (2016) 363:399-409

DOI 10.1007/s00441-015-2262-0

The original version of this article inadvertently contained mistake.

Dr. Jun Yang (same affiliation as Dr. K.S. Lee and Dr. B.D. Hammock, remarked as 2) should be the second author of the paper but was accidently removed.

Also, the following sentence should be added at the end of the acknowledgments: "NIH CounterAct U54 NS079202 and K.S.S.L. is partially supported by the NIH Pathway to Independence Award from NIH/NIEHS (1K99ES024806-01)".

The online version of the original article can be found at http://dx.doi.org/ 10.1007/s00441-015-2262-0.

Jian-Xin Jiang

hellojjx@126.com

$\triangle$ Cha-Xiang Guan

guanchaxiang@csu.edu.cn

1 Department of Physiology, Xiangya School of Medicine, Central South University, Changsha, Hunan 410078, People's Republic of China

2 Department of Entomology and the UC Davis Cancer Center, University of California Davis, Davis, CA 95616, USA

3 Department of Neurology, University of Texas Medical Branch, Galveston, TX 77555, USA

4 State Key Laboratory of Trauma, Burns, and Combined Injury, Research Institute of Surgery, Daping Hospital, Third Military Medical University, Chongqing, Sichuan 400042, People's Republic of China 